\title{
Spinal Nephroblastoma in a Miniature Dachshund
}

\author{
Tetsuya NAKADE ${ }^{1)}$, Aika INOUE ${ }^{1)}$, Hitoshi SHIMAZAKI ${ }^{1)}$, Kenjirou MIYOSHI ${ }^{1)}$, Nobuyoshi TAKEUCHI ${ }^{1)}$, \\ Tsuyoshi KADOSAWA ${ }^{2)}$, Yuhko AKIHARA ${ }^{3)}$, Hiroyuki TANIYAMA ${ }^{3)}$ and Osamu ISHIDA ${ }^{4}$
}

\begin{abstract}
${ }^{1)}$ Sections of Veterinary Diagnostic Imaging and Interventional Radiology, ${ }^{2)}$ Veterinary Oncology and ${ }^{3)}$ Veterinary Pathology, School of Veterinary Medicine, Rakuno Gakuen University, 582 Bunkyodai Midorimachi, Ebetsu, Hokkaido, 069-8501 and ${ }^{4}$ Kitasapporo Animal Hospital, Kitaku, Sapporo, Hokkaido, 011-0035, Japan
\end{abstract}

(Received 26 June 2006/Accepted 7 September 2006)

ABSTRACT. A 2-year-old castrated miniature Dachshund dog was presented to the Rakuno Gakuen Veterinary Teaching Hospital for diagnosis of progressive hindlimb paresis and ataxia. There was no thoracolumbar intervertebral disk hernia and magnetic resonance imaging revealed an intramedullary spinal cord lesion at the ninth and tenth thoracic vertebrae. Following surgical excision of the neoplasm, there was minor amelioration of neurological signs, but forelimb function was not recovered. The extracted tumor was histopathlogically diagnosed as spinal nephroblastoma.

KEY WORDS: canine, magnetic resonance imaging (MRI), spinal nephroblastoma.

Nephroblastoma of the spinal cord is a rare neoplasm in young dogs. Myelography for diagnosis of spinal neoplasm has been described $[3,4,11]$, but more recently, there have been some case reports on the magnetic resonance imaging (MRI) features of nephroblastoma in dogs $[5,8]$. This paper describes the MRI, surgical treatment and histopathology of canine spinal cord nephroblastoma with progressive hindlimb paresis and ataxia.

A 2-year-old castrated miniature Dachshund dog was referred to the Rakuno Gakuen Veterinary Teaching Hospital for diagnosis of progressive hindlimb paresis and ataxia. The dog had sudden onset of left hindlimb lameness 3 weeks earlier. He was treated with oral prednisolone and intravenous methyrprednisolone, but there was no improvement for three weeks.

Right hindlimb paresis appeared during the fourth week, indicating that the bilateral hindlimb paresis and ataxia were progressive. Neurological examination revealed proprioceptive, hopping and placing deficits of the left and right hindlimbs. Patellar and pedal reflexes were normal or slightly asthenic. The forelimb was normal and the hindlimb was positive for upper motor neuron sign. Based on clinical neurologic examination, an upper motor neuron third thoracic to third lumbar spinal cord lesion was suspected.

Survey radiographs of the entire spine were normal. The dog was anesthetized and imaged in dorsal recumbency using a 0.2-Tesla MR scanner (Signa Profile Open Sprit, GE Yokogawa Medical Systems, Tokyo, Japan) with a surfacearray human extremity coil. T2-weighted and T1-weighted images were obtained in the transvervse and sagittal planes at slice thicknesses of 2.7 and $3.5 \mathrm{~mm}$.

T2-weighted, midline sagittal slices revealed an abnormal region in the spinal cord at the ninth and tenth thoracic vertebrae (Fig. 1). The spinal cord lesion was isointense relative to normal spinal cord on T1-weighted MRI (Fig. 2). On enhanced T1-weighted midline sagittal images after intrave- nous administration of the meglumine gadopentetate $(\mathrm{Gd}$; Magnebisto Nihon Shering Inc., Osaka. $0.1 \mathrm{mmol} / \mathrm{kg}$ body weight), the lesion was enhanced and observed at the ninth and tenth thoracic vertebrae. The enhanced lesion was 27 $\mathrm{mm}$ in length (Fig. 3). On transverse images, the mass occu-

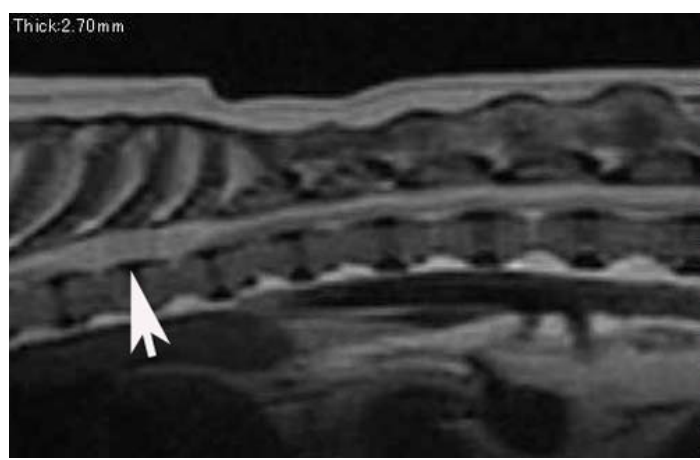

Fig. 1. T2-weighted midline sagittal slices revealed an abnormal region in the spinal cord at the ninth and tenth thoracic vertebrae (arrow).

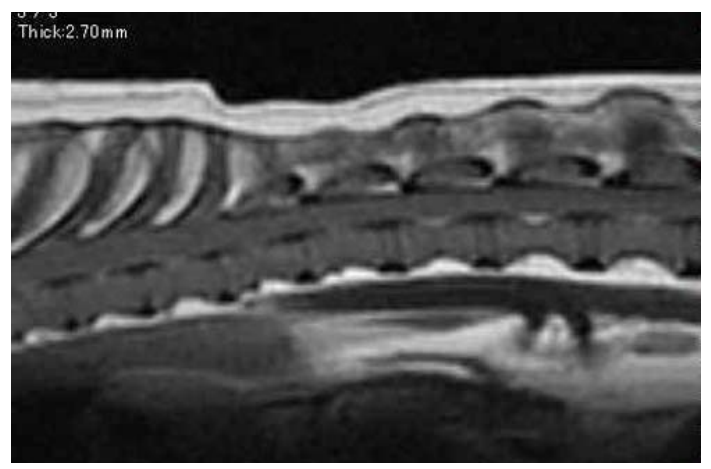

Fig. 2. T1-weighted thoracolumber sagittal slices. Spinal cord lesion is isointense on T1-weighted MRI. 


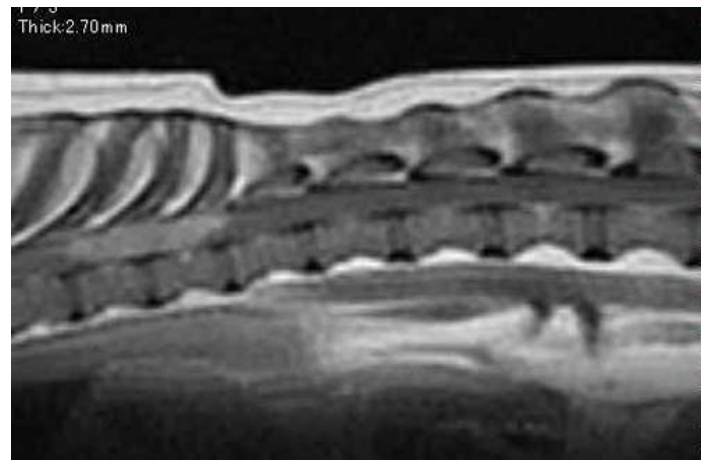

Fig. 3. T1-weighted images with meglumine gadopentetate (Gd) enhanced the lesion at the ninth and tenth thoracic vertebrae.

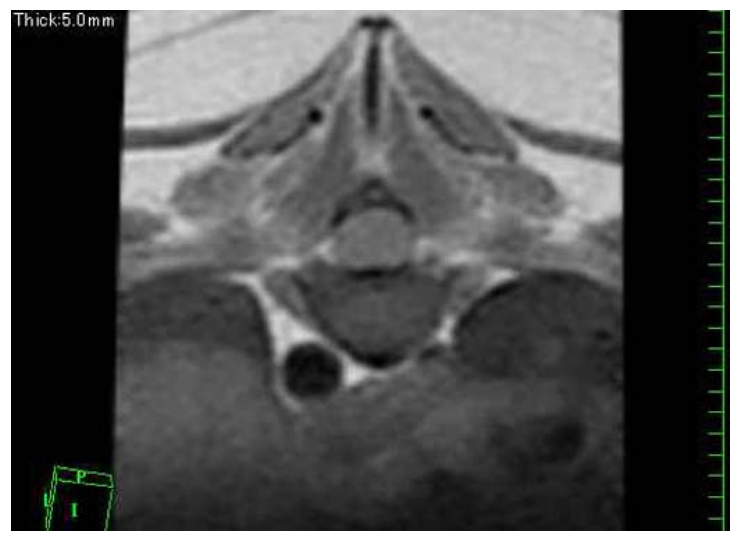

Fig. 4. T1-weighted transverse images with $\mathrm{Gd}$ at the tenth thoracic vertebrae revealed the majority of the intramedullary spinal cord lesion.

pied almost the entire cross-sectional area of the spinal cord at the ninth and tenth thoracic vertebrae of the intramedullary spinal cord lesion (Fig. 4). A tentative diagnosis of spinal neoplasia, strongly suspected to be nephroblastoma or meningioma, was made based on the young age of the dog and these examinations.

Dorsal laminectomy of the ninth and tenth thoracic vertebrae was then performed. A $25 \times 10 \mathrm{~mm}$, reddish area of discoloration was visible through the dura. The dural membranes were incised in using a surgical knife. There was no adhesion between the mass and dura. Spinal cord swelling was observed and the border between the mass and spinal cord was unclear. The mass was dissected from the parenchyma using a combination of gentle traction, as well as blunt and sharp dissection. The dura was closed with 5-0 nylon sutures.

Histologically, the tumor was composed of tubules and acini lined with tall epithelial cells having little cytoplasm (Fig. 5). These epithelial cells were occasionally invaginated into a central lumen, resembling embryonic glomeruli (Fig. 6). These structures were partially encompassed by loose mesenchymal cells or solid sheets of ovoid cell. Mito-

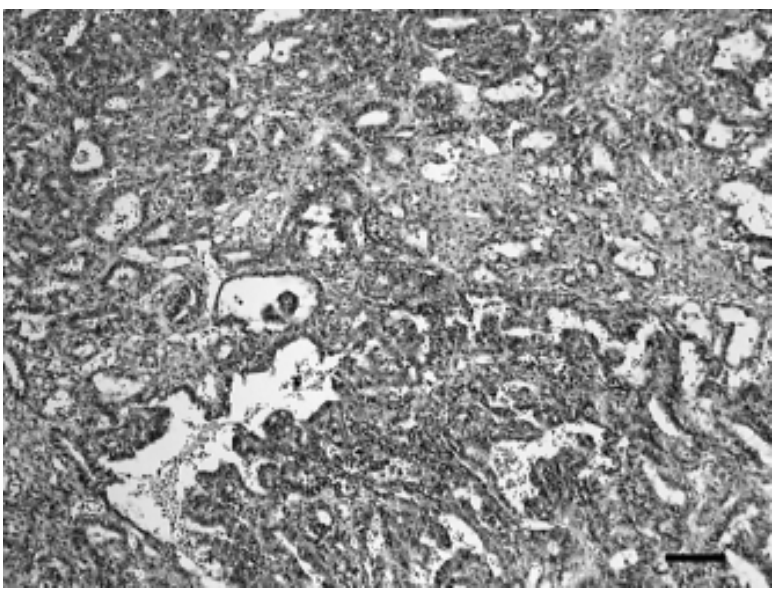

Fig. 5. Irregularly shaped tubular and acinus structure lined with tall epithelial cells stained by HE. Bar $=50 \mu \mathrm{m}$.

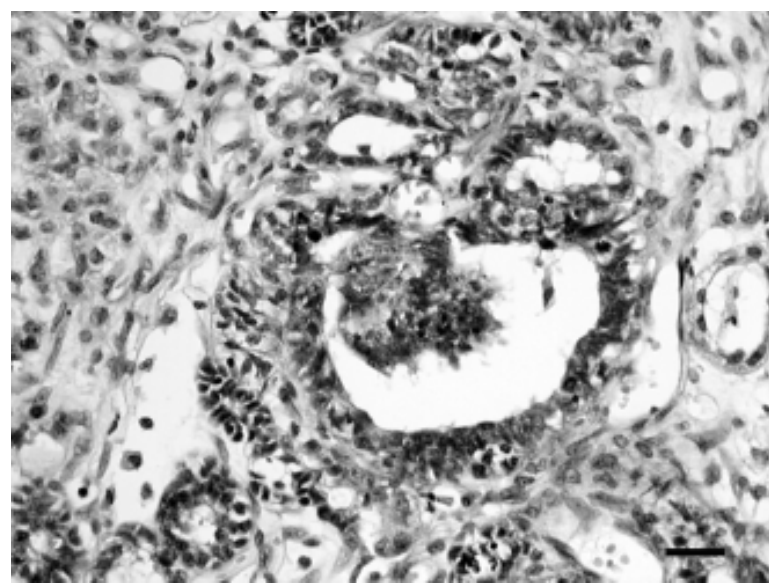

Fig. 6. Higher magnification illustrates invagination of epithelial cells, resembling embryonic glomeruli. HE staining. Bar=30 $\mu \mathrm{m}$.

sis was observed in 1-2 cells per high-power field.

Following surgical excision of the neoplasm, there was minor amelioration of the neurological signs, but intact forelimb function was not recovered. No recurrence has been observed in the ninth and tenth thoracic vertebrae on MRI at 8 post-operative months.

Spinal nephroblastoma typically occurs in dogs between six months and three years of age $[2,4,6,7,9,10]$, but has been described in dogs as old as 7 years [1], and is most commonly found between the tenth thoracic and second lumber vertebrae. It is most often seen in medium to large dogs, and German shepherds have a particularly high incidence. Unusually, the present case was a Miniature Dachshund, and thus spinal nephroblastoma should also be considered in smaller breeds.

The tumors are thought to arise from embryonic renal tissue that becomes entrapped in the dura of the spinal canal during fetal development [8]. Tumor lesions are commonly 
described as intradural and extramedullary, but two cases have been described as intramedullary $[4,8]$, similarly to the present case reported here. The amelioration in clinical neurological signs was mild in this case. On the other hand, another report described a dog recovering from nephoroblastoma for fifty-one weeks, after which local recurrence was observed [8]. We believe that differences in recovery depend on the degree of the localization in the intramedullary region. Little healthy spinal cord remained in the present case, and thus neurological signs did not improve markedly.

There is a tendency to diagnose hernia in chondralloplasic dog breeds with progressive hindlimb paresis and ataxia; however, spinal nephroblastoma should be considered in the differential diagnosis.

\section{REFERENCES}

1. Clark, D. M. and Picut, C. A. 1986. J. Am. Vet. Med. Assoc.
189: 1330-1331.

2. Ferrett, A., Scanziani, E. and Colombo, S. 1993. Pro. in Vet. Neurol. 4: 84-87.

3. Jeffery, N. D. and Phillips, S. M. 1995. J. Small Anim.Prac. 36: 553-557.

4. Macri, N. P., Van Alstine, W. and Coolman, R. A. 1997. J. Am. Anim. Hosp. Assoc. 33: 302-306.

5. McConnell, J. F., Garosi, L. S., Dennis,R. and Smith, K. C. 2003. Vet. Radio. and Ultrasound. 44: 537-541.

6. Moissonnier, P. and Abbott, D.P. 1993. J. Am. Anim. Hosp. Assoc. 19: 397-401.

7. Pearson, G. R., Gregory, S. P. and Charles, A. K. 1997. J. Comp. Pathol. 116: 321-327.

8. Sale, C. S. H., Skerritt, G. C. and Smith, K.C. 2004. J. Small Anim.Pract. 45: 267-271.

9. Summers, B.A., deLahunta, A., McEntee, M. and Kuhajda, F. P. 1988. Acta Neuropathologica 75: 402-410.

10. Tamke, P. G. and Foley, G. L. 1987. Can. Vet. J. 28: 606-608.

11. Vaughan-Scott, T., Goldin, J. and Nesbit, J.W. 1999. J. S. Afr. Vet. Assoc. 70: 25-28. 